\title{
Emerging infections: mimickers of common patterns seen in dermatopathology
}

\author{
Francisco G. Bravo'
}

Received: 30 June 2019 / Revised: 14 October 2019 / Accepted: 14 October 2019 / Published online: 4 November 2019

(c) The Author(s), under exclusive licence to United States \& Canadian Academy of Pathology 2019

\begin{abstract}
The following discussion deals with three emerging infection diseases that any dermatopathologist working in the northern hemisphere can come across. The first subject to be dealt with is gnathostomiasis. This parasitic disease is produced by the third larvarial stage of the parasite that in most patients is associated with the ingestion of raw fish. Epidemiologically, it is most commonly seen in South East Asia, Japan, China, and the American continent, mainly in Mexico, Ecuador, and Peru. Nowadays, the disease is also seen in travelers living in the developed countries who recently came back from visiting endemic countries. The disease produces a pattern of migratory panniculitis or dermatitis with infiltration of eosinophils in tissue. The requirements for making the diagnosis are provided, including clinical forms, common histological findings on skin biopsy as well as the use of ancillary testing. Buruli ulcer, a prevalent mycobacterial infection in Africa, is described from the clinical and histopathological point of view. The disease has been described occasionally in Central and South America as well as in developed countries such as Australia and Japan; Buruli ulcer has also been described in travelers returning from endemic areas. Clinically, the disease is characterized by large, painless ulcerations with undermined borders. Systemic symptoms are usually absent. Classical histological findings include a particular type of fat necrosis and the presence of abundant acid fast bacilli in tissue. Such findings should raise the possibility of this disease, with the purpose of early therapeutically intervention. Lastly, the infection by free living ameba Balamuthia mandrillaris, an emerging condition seen in the US and Peru, is extensively discussed. Special attention is given to clinical and histological characteristics, as well as to the clues for early diagnosis and the tools available for confirmation.
\end{abstract}

\section{A migratory eosinophilic panniculitis and/or dermatitis}

Many times, in dermatopathology, one encounters an eosinophilic perivascular dermatitis. Insect bite reactions, eczematous processes, drugs reactions, urticaria and urticarial reactions are all among the clinical entities to be considered in the differential diagnosis of eosinophilic infiltrates. The eosinophil is an inflammatory cell that, besides being involved in allergic processes, also has antiparasitic properties. Its presence in the dermis begins as a perivascular infiltrate, superficial, or superficial and deep, to later evolve into an interstitial and wedge shaped

Francisco G. Bravo

fbravopuccio@gmail.com

1 Universidad Peruana Cayetano Heredia, Hospital Cayetano Heredia, Lima, Peru eosinophilic infiltrate. That is the classical pattern seen in an insect bite reaction and similar reactions, such as scabies. Sometimes the density of the infiltrate is what makes the difference. Urticaria and urticarial reaction are characterized by a scarce superficial perivascular infiltrate of eosinophils, and minimal edema of the papillary dermis. An edematous papillary dermis and many eosinophils located in that precise area are more suggestive of the urticarial stage of pemphigoid.

But, what if there is a predominant perivascular and interstitial, superficial and deep infiltrate, of the same density in the upper or lower dermis, rather than wedge shaped, with no epidermal changes? Or what if the maximal density is located in the lower reticular dermis, in the area between the dermis and the panniculus, or mainly in the panniculus in a lobular pattern? Those patterns should raise the possibility of a mobile parasite, especially when the clinical history is either a plaque or a nodule that migrate with the passing of the days. 
Factors such as the event occurring in specific endemic areas of the world, or a history of traveling to endemic areas, accompanied by the history of eating raw fish, increase the possibility of Gnathostomiasis.

\section{Gnathostomiasis}

\section{Introduction}

Gnathostomiasis is a parasitic infection, caused by the migration of the third larvarial stage (L3) of parasites of the Gnathostoma genus. They are nematodes belonging to the order Spirurida. Thirteen species have been described, with five of them been identified as causing disease in humans. G. spinigerum is the most common of all, especially in Asia $[1,2]$. G. binucleatum seems to be responsible for cases seen in Mexico, Ecuador, and Peru [3].

\section{Epidemiology and etiopathogenesis}

There are certain areas of the world that are considered endemic for Gnathostomiasis such as South East Asia, Japan, China, Korea, Mexico, and South America, mainly Ecuador and Peru [2]. The disease is seen also in returning travelers, such as residents in North America and Europe $[4,5]$ who consumed raw fish dishes while they were visiting endemic areas. There are also new cases of gnathostomiasis coming from countries not considered endemic, such as Brazil [2]. In recent described Brazilian cases, the only risk factor was that the people affected had consumed raw fish while fishing and camping during short trips to the interior of the country. It is possible that new species native to other areas and other countries are yet to be discovered as the disease becomes better known and recognized.

The infection is clearly connected to the consumption of raw freshwater fish, but also to the consumption of meat from different animals, and to drinking contaminated water. An interesting exception for the freshwater fish consumption are the cases seen in Peru [6], where the raw fish consumed in the form of ceviche is always from the Pacific ocean; the fish species acting as the source of the infection is unclear, but the cases are clearly associated with consumption of expensive fish in the form of ceviche.

The adult form of Gnathostoma worms have cats and dogs as definitive hosts; in the wilderness, big felines play that role. In the case of domestic animals, these live in the margins of rivers and estuaries, near the ocean, or in brackish waters; the domestic animals usually feed on rotten fish discarded by local fishermen. Such a fish carries the third larvarial stage of Gnathostoma. When ingested by the domestic animals, the larva infects the cat, dog or any local susceptible feline species, to finally form a nest in their gastric mucosae. The adult female and male reach a reproductive stage, and the fertile female start to eliminate parasitic eggs through the feces of the host. The eggs reach a freshwater environment, and evolve into a first larvarial stage, ingested by copepods of the genus Cyclops, maturing into a second larvarial stage, and then into a third larvarial state in the muscular mass of either a fish, a bird or any other animal susceptible to be part of the food chain; the L3 larvae will end up been eaten by the domestic cats and dogs, allowing the worm to complete the natural cycle. Human beings are accidentally infected when they consume raw fish, avian, or reptilian meat carrying the L3 larvae.

Once a human ingests the $\mathrm{L} 3$ form, as soon the piece of contaminated meat reaches the gastric space, the worm is liberated and, by following its natural instincts, perforates the gastric mucosa and starts its wandering migration inside the peritoneal cavity. The moment of perforation of the gastric mucosa may happen asymptomatically or may produce pain mimicking an acute abdomen. The worm can also choose to go into the liver, causing biliary symptoms, or to the pleural space and even the lungs, causing respiratory symptoms. If the peritoneal perforation to the panniculus takes place without clinical symptoms (most cases), the L3 form will eventually end up in the subcutaneous tissue. In an unknown territory such as the human body, 2-4 weeks after the ingestion of the L3 larva, the larva will start a migratory course, causing a pattern of a deep panniculitis or a more superficial pattern mimicking cutaneous larva migrans. The episode may last 2 weeks, after which it will disappear spontaneously. The course can be chronic and can last for years, with active migration alternating with dormant periods. While the adult forms measure 15 to $35 \mathrm{~mm}$ in length, the invasive L3 form measures up to $12.5 \mathrm{~mm}$ in length and $1.2 \mathrm{~mm}$ in diameter [1]. The size of the invasive larva is such that, when they emerge spontaneously, it can be seen with the naked eye.

Identifiable characteristics that may separate each species include the shape of the body, the number of rows of hooks at the cephalic end, the number of hooks in each row, the character of the spines which cover the body, and the extent to which the body is covered by spines, which vary according to the species [1]. The cephalic pole of the worm looks very similar to the frontal pole of a tunnel drill machine, making it easy to imagine the act of perforation through the tissue.

\section{Clinical manifestations}

The most common manifestations of the infection are localized on the skin. The classical and most common 


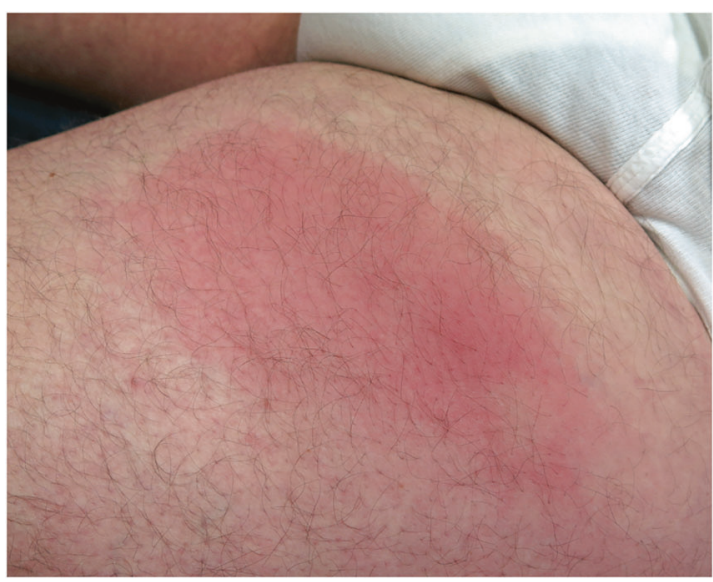

Fig. 1 Plaque-like lesion of gnathostomiasis

manifestation is the formation of an ill-defined plaque (Fig. 1) or a nodule on any skin location, although most commonly seen on the abdominal region, chest, or extremities. Distal locations on extremities, such as hands and feet, may occur, and are commonly complicated with marked edema. The patient may complain of pain or itching or may go asymptomatic. More risky areas include the head, because of the potential to invade the central nervous system (CNS), and especially around the eyes, because of the potential to invade the ocular globe. The natural history will be toward spontaneous resolution of the skin involvement after a few days or weeks. However, after this apparent resolution, the same manifestations will reappear as a new episode, located at a short distance from the original site, giving an idea of migratory panniculitis or migratory infiltrative dermatitis. Sometimes the surface of the affected area may look like "peau d'orange". Patients also may present with a clinical picture identical to the serpiginous pattern seen in cutaneous larva migrans; both presentations may occur sequentially. As opposed to true cutaneous larva migrans, the location may seem unusual, not on the feet or the buttocks, but rather on the abdomen, chest, breasts, or the head. There is a third form of cutaneous presentation of gnathostomiasis, called the furuncle-like pattern; in such cases, the L3 comes so close to the surface, that it looks like either a small papule or a pustule [2]. In this situation, the worm is located perpendicular to the surface of the epidermis; then, it will be more likely to emerge spontaneously or may be extracted with a simple punch biopsy. In our experience, this form will be seen more commonly after the patient has already received the antiparasitic therapy [7]. All clinical forms may be combined in the same patient (Fig. 2). Most patients are affected by a single worm.

Visceral involvement to other organs such as pleural space, lung tissue, the gastrointestinal system, and the genitourinary system may occur; the worst-case scenery occurs if the parasite penetrates the eye or the CNS.

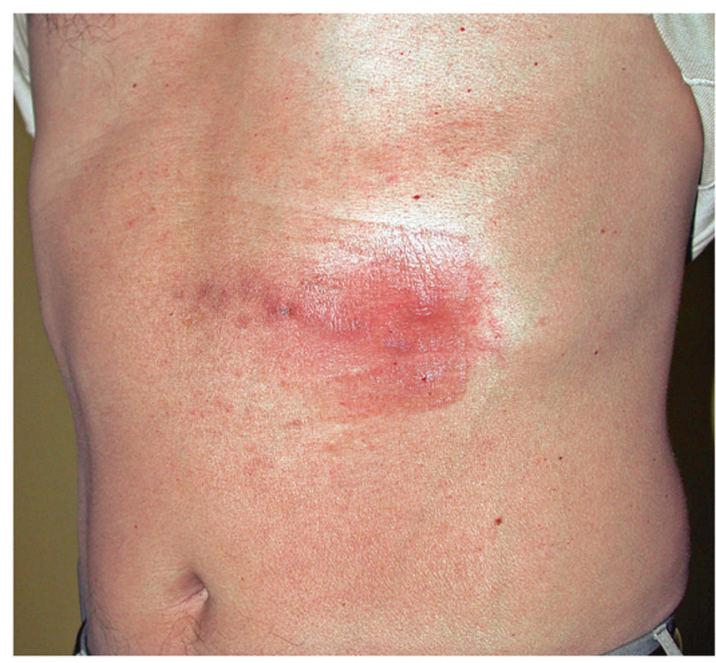

Fig. 2 Mixed plaque-like lesion and cutaneous larva-migranslike lesion

\section{Histopathology}

The most common finding when a biopsy is taken from a cutaneous lesion will consist of different degrees of infiltration by eosinophils. In the case of deep-seated nodules or ill-defined plaques of panniculitis, the biopsy should be done sampling deep tissue, down to the panniculus. The most common finding is a lobular infiltration by abundant eosinophils (Fig. 3), but in some cases the numbers of eosinophils in the fat are scarce. In cases where the clinical lesion is more superficial, the infiltrates occupy mostly the reticular dermis (especially when the clinical is of cutaneous larva migrans). Again, different number of eosinophils can be seen; if they are abundant, eosinophilic changes can be seen in collagen fibers, mimicking the flames figures classically associated with Well's cellulitis. The eosinophils may be only interstitial in the dermis. When the clinical findings are those of "peau d'orange", there is a significant papillary dermal edema with great number of eosinophils. The infiltrates may also contain some lymphocytes but neutrophils, histiocytes, or giant cells are rarely present.

The biopsy of a papular or pustular lesion (so called furuncle-like lesion), especially when they appear as a consequence of the administration of antiparasitic medication (either albendazole or ivermectin), may show some transversal sections of the parasite [2]. The chances of seeing the parasite increase greatly depending on the time of biopsy (especially after treatment has been given) [7] (Fig. 4). The parasite is usually located in the reticular dermis. Its aspect is usually oval or circular on transversal sections and it has spiny processes in the surface [2]. The anterior segment shows a round mouth, followed by an esophagus with a thick muscular wall, whereas the posterior segment may show a pigmented intestinal tract (Fig. 4). The 
presence of the gastrointestinal tract allows its identification as a nematode [2]. The diameter can be measured with a micrometer; it is usually between 0.4 and $0.6 \mathrm{~mm}$ in diameter. Its size and its location (reticular dermis or subcutaneous tissue) allow the observer to clearly distinguish it from the Ancylostoma sp. and other nematodes causing

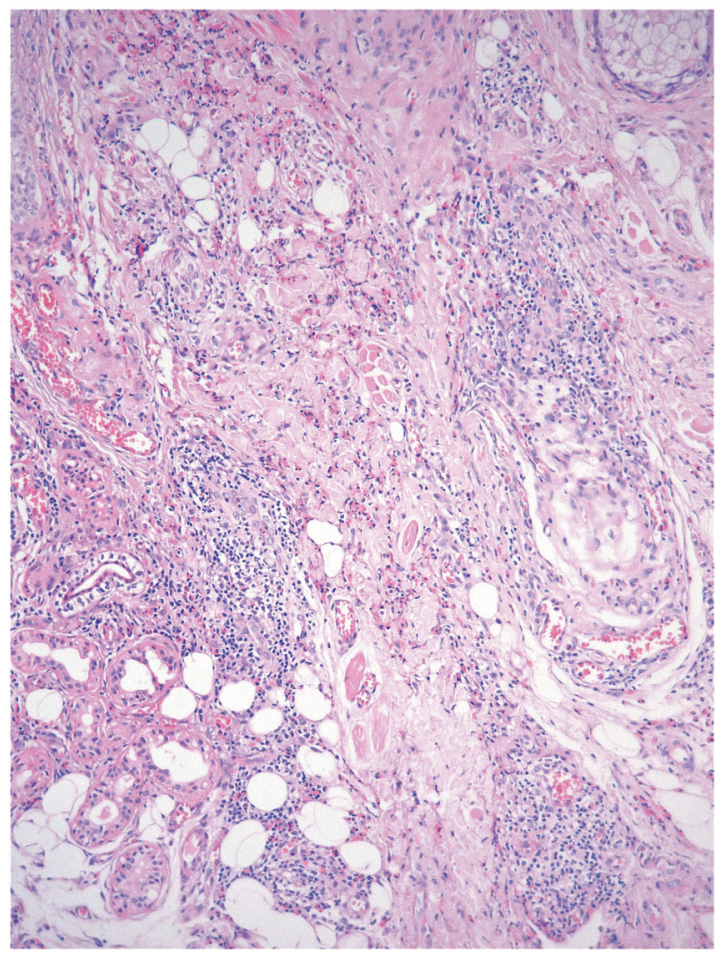

Fig. 3 Typical biopsy of gnathostomiasis: heavy inflammatory infiltrate of eosinophils and lymphocytes deep in the reticular dermis and extending into the fat. $\mathrm{H} / \mathrm{E}, \times 20$ cutaneous larva migrans. The migrating larva of the last group of worms is quite smaller and travels inside the epidermis. The filariform larva of Strongyloides sp. travels in the dermis but its size is also small, comparable to Ancylostoma sp., and much smaller than Gnathostoma. Another worm that can be found in a migrating pattern is the Sparganum, the invasive larvarial form of Spirometra $s p$. However, the larva of sparganosis is easy to differentiate, because it is a cestode, with no intestinal tract but feeding channels and calcified bodies.

\section{Diagnosis}

The diagnosis is made mostly on clinical and histological grounds, based on the history of consumption of raw fish in various gastronomic preparations (ceviche, sashimi, or sushi), a clinical migratory deep-seated lesion in the subcutaneous tissue, a cutaneous pattern of cutaneous larva migrans in a location beyond the lower extremities and buttocks, and also the combination of both patterns. Although rare, the furuncle-like pattern is the one that carries the highest chance of seeing the parasite in tissue cuts. The patient with suspicion of gnathostomiasis should also have evidence of peripheral eosinofilia $(50-70 \%$ of patients) [6] and tissue eosinophilia in the skin biopsy. Absence of peripheral eosinophilia, however, does not exclude the diagnosis.

Seeing the parasite in the skin biopsy is a rare event but certainly confirmatory (16\% of cases) [6]. Other tests include serology testing against a specific antigen of L3, of $24 \mathrm{kDa}$ (considered by many as the most specific, but not
Fig. 4 The L3 larvae of gnathostomiasis as it was isolated from a pustule of a patient after therapy. Look at the round mouth followed by the muscular esophagus (clear in color) and the pigmented intestine (upper figure). The lower figure on the left is the esophagus and the one on the right is the intestine as seen in histological cuts from different patients

readily available), an ELISA testing against L3 immunoglobulin $\mathrm{IgG} 2$, immunoblotting against the $24 \mathrm{kDa}$ antigen, and a recent western blot method directed to L3 from G. binucleatum isolated larva from Ecuador [3]. The last study has demonstrated that the positivity of the test may be species dependent.

\section{Prognosis}

The prognosis of the cutaneous disease is good with adequate therapy with albendazole or ivermectin. Cases with eye involvement and CNS involvement have a more guarded prognosis.

\section{A chronic ulceration with undermined borders and a specific pattern of necrosis with lack of inflammatory cells}

In everyday dermatopathology, the evaluation of chronic skin ulceration by clinicians often includes a skin biopsy of the borders and the bottom of the lesions. The most common cause of ulceration in lower extremities is venous ulceration, but other causes include neoplasia and infections. Also, most complex vascular inflammatory diseases, including forms of immune complex vasculitis and granulomatous vasculitis, can be evaluated with a good biopsy. But what if the main finding is a particular type of necrosis, seen in the subcutaneous tissue, in the absence of a significant inflammatory infiltrate, and in the presence of an abundant collection of acid fast bacilli? Then, in the appropriate clinical context, other diagnoses should be considered, including diseases that may not be commonly seen in developed nations, but that represent a significant health problem of worldwide dimension. That is the case of Buruli ulcer.

\section{Buruli ulcer}

\section{Introduction}

Buruli ulcer may sound like an exotic disease to the average pathologist, but in terms of worldwide public health, it is a disease of great importance. It is caused by Mycobacterium ulcerans, a unique mycobacteria capable of causing disease by producing a toxin, known as mycolactone. After tuberculosis and leprosy, it is the third most common mycobacterial infection around the world, and an important cause of disability in Central Africa with occasional cases in South America and Papua-New Guinea, and even affecting people in some developed countries such as Australia and Japan.

\section{Epidemiology and etiopathogenesis}

Buruli ulcer has been reported in 33 countries, with 3000-5000 patients reported yearly [8]. Initially described in Australia as Bairnsdale ulcer in the 1930's, it was later described in the Buruli valley in Uganda. Nowadays it is mainly reported in developing countries in West Central Africa. Countries with the highest burden of disease include Côte d'Ivoire, Ghana and Benin. In West Africa, the main population affected is children between the age of 5-15 years. In more temperate climates, such as Australia and Japan, the disease affects mainly adults. In the American continent, cases have been described in French Guyana, Suriname, Peru, Mexico, and Bolivia $[8,9]$.

The agent, Mycobacterium ulcerans, is a slow growing mycobacterium, taking 4-12 weeks to appear in culture. The bacterium is microaerophilic, with a temperature requirement of $28-33^{\circ} \mathrm{C}$, much lower than that for other mycobacteria $\left(37^{\circ} \mathrm{C}\right)$. Morphologically, it is similar to Mycobacterium tuberculosis, but genetically, it is very closed to M. marinum. Some researchers have hypothesized that $M$. ulcerans evolved from an M. marinum that acquired the capacity of synthesizing the mycolactone toxin by an insertion of a plasmid. Similar to M. marinum, it is isolated commonly from water sources and aquatic insects, fish, and animals. Rather than transmission through vectors, the infection is supposedly acquired directly from the environment by minimal trauma or injuries caused by scratching insect bites.

In regard to its bacteriology, two characteristics make it a particular mycobacteria: the presence of the plasmid pMUM001, responsible for the mycolactone production, and the presence of multiple copies of IS2404 (more than 200) in its genome. The expansion of IS2404 disrupted coding regions and/or promoter regions; as a result, $M$. ulcerans has more than 700 pseudogenes compared with less than 100 seen in M. marinum. [8].

Comparative analysis of whole genomic sequences of $M$. ulcerans and other mycolactone-producing mycobacteria have showed two genetic lineages, the "classical" and the "ancestral". The classical lineage includes the cases from Africa and Australia, whereas the ancestral lineage includes the cases seen in Japan, China, Mexico, Suriname, and French Guyana [8].

The pathogenesis capacity of the bacterium is related to the production of the mycolactone toxin. This is a polyketide-derived macrolide that has cytotoxic, immunosuppressive, and anesthetic properties, which explains the special necrosis seen on histology, the lack of inflammatory reaction in the tissue, the absence of systemic symptoms (fever and malaise), and the lack of pain in the presence of large necrotic ulcerations. 


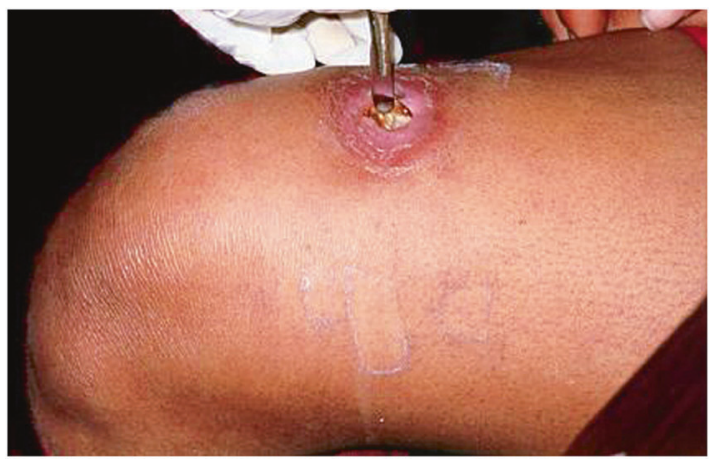

Fig. 5 Buruli ulcer in the knee area. The patient came from the Peruvian Amazonia. Notice the characteristic undermining

\section{Clinical findings}

Patients in Africa, as mentioned before, are mainly children, and in a lesser proportion, adults that, due to their recreational or occupational activities, are in contact with water sources. In other endemic areas, such as Australia, Japan, and Peru, adults are mainly affected [8,9]. The lesion may start most commonly as a papule or a nodule in the African cases. Some cases are diagnosed at those early stages. However, many lesions evolve into large abscesses that end up opening into an ulcer. The diameter of the ulcer opening stays the same, but at the limit between deep dermis and subcutaneous tissue, the area of necrosis continues to grow horizontally, in an undermining fashion (Fig. 5). This undermining is the most important clinical characteristic of the disease. Sometimes, rather than a single ulceration, a whole extremity becomes edematous. When an incision is made, all the underlying subcutaneous tissue below the unaffected epidermis is involved by the necrotic process. Commonly affected areas include the extremities and the face, but the disease may be located anywhere. The process has the potential to extend into the underlying bone structures, causing osteomyelitis. Early diagnosis may allow an early treatment, avoiding the marked deformities and disabilities linked to advance disease. Buruli ulcer does not kill, but it is an important cause of morbidity because of scarring and fibrosis.

\section{Histopathology}

The classical findings in Buruli ulcer are mainly located at the level of the deep dermis and the subcutaneous tissue. If the biopsy is limited to the surface of the ulcer, the finding may be unspecific, similar to any other ulcer. There will be granulation tissue and neutrophils. The most important tissue to evaluate is the fat tissue surrounding the ulceration. The telling finding is the presence of fat necrosis (Fig. 6). The fat cells enlarge, losing their nuclei

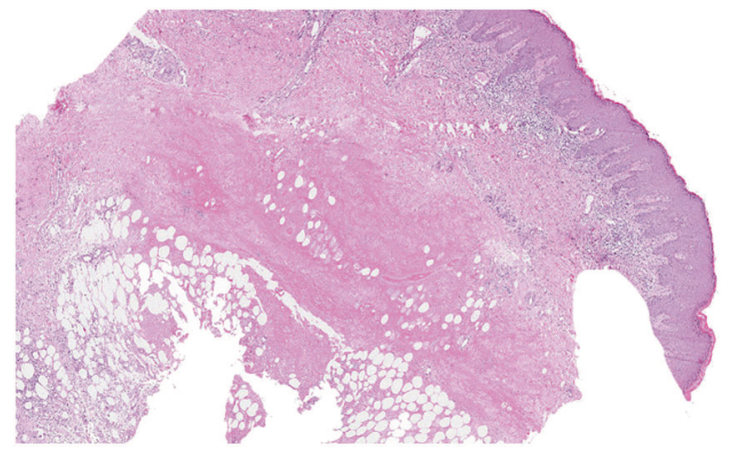

Fig. 6 This is a biopsy of the tissue surrounding the ulceration. At the center, there is necrosis in deep dermis and subcutaneous fat. $\mathrm{H}$ / E, $\times 100$

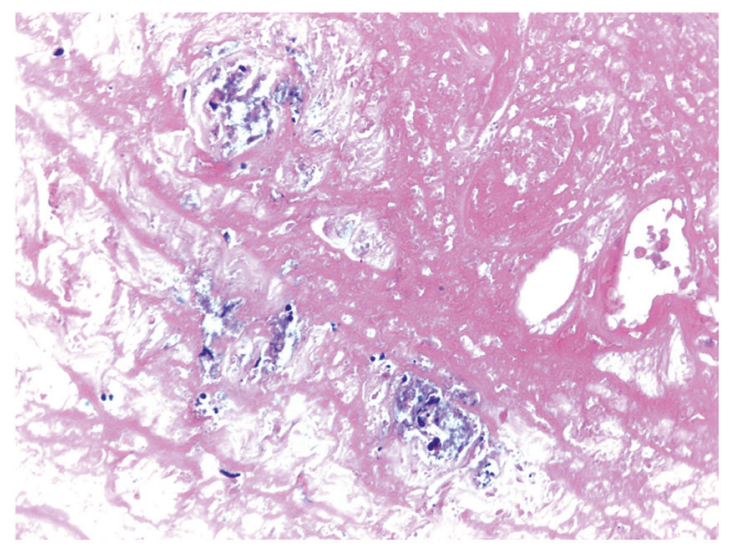

Fig. 7 Fat and dermal necrosis (ghost fat cells). The bluish material are bacilli. H/E, $\times 400$

but preserving their membrane, giving the appearance of "ghost" fat cells (Fig. 7). Minimal or no inflammatory cell infiltrate is present, but neutrophils have been described associated with high bacterial load in the areas of necrosis [10]. Areas of bluish material in the periphery of the fat necrosis represent clumps of bacilli, better distinguished with a Ziehl-Neelsen stain (Fig. 8). A particular observation is that the degree of the staining may be variable, with some bacteria absorbing the $\mathrm{Z}-\mathrm{N}$ dye better than others. The frequency with which bacilli are found in the biopsy varies. It has been estimated that around 30-40\% of cases with necrosis will demonstrate the presence of bacilli in special stains [11]; however, some cases that are highly suspicious clinically may not show the presence of bacteria in tissue. A second biopsy may be helpful in finding the bacteria. Granulomas have been described at the periphery of the area of necrosis and in cases with prolonged evolution. Vascular changes from necrosis to thrombosis can occasionally be seen. In later states fibrosis may predominate. The epidermal changes are not specific: ulceration, psoriasiform hyperplasia, and pseudoepitheliomatous hyperplasia have all been described. 


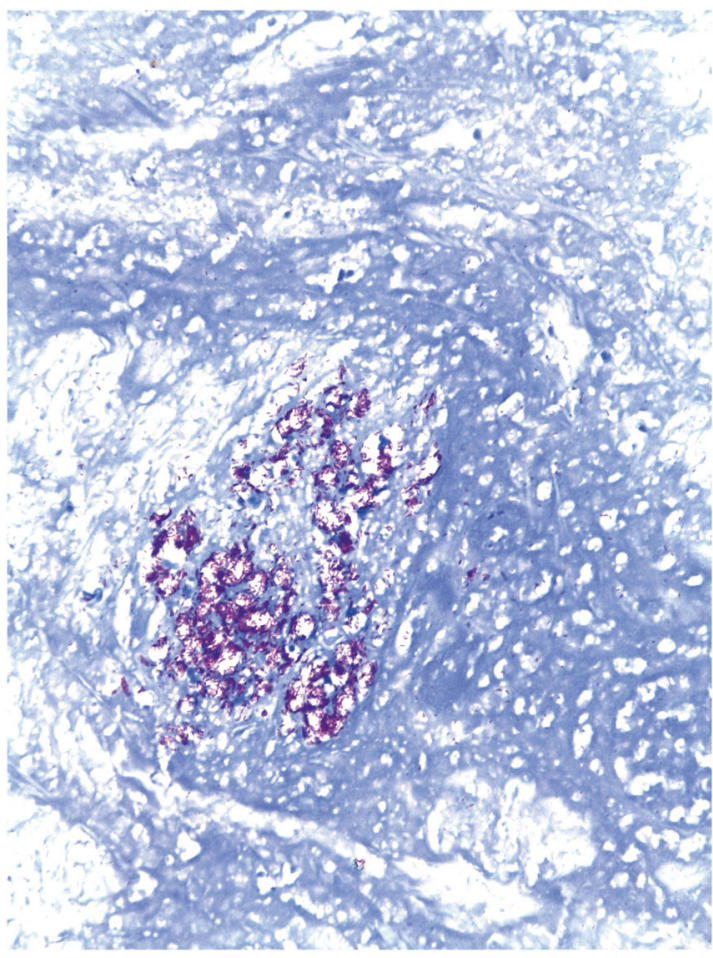

Fig. 8 Same area when stained with Ziehl-Neelsen: it is possible to identify the large number of mycobacteria (Mycobacterium ulcerans) in clumps

\section{Diagnosis}

The diagnosis requires a clinical history of exposure to water sources. The disease is well recognized in endemic areas but also has been diagnosed in travelers to countries were the disease has been described [12-15].

An asymptomatic ulcer with undermined borders, and no systemic symptoms, is the most important clinical clue. However, the nodular and plaque form do exist, and ulceration may be absent.

If Buruli ulcer is in the differential diagnosis, a biopsy should be done, and should include the subcutaneous tissue surrounding the ulceration.

Necrosis of the fat tissue and the absence of massive infiltrate should raise the possibility of Buruli ulcer. The bacilli are acid-fast positive; in cases with a positive the bacilli are usually abundant, either as clumps or in a diffuse distribution. Such number of bacilli are only seen in the skin lesions of Leprosy, Mycobacterium avium intracellulare infection in HIV, and tuberculosis in immunosuppressed patients. However, an absence of Ziehl-Neelsen bacilli in a biopsy does not rule out Buruli ulcer and a second biopsy may be required.

Regarding microbiology studies, direct smear or touchprep may be positive but nonspecific, but in the context of clinical findings they could be helpful. The culture can identify viable bacteria. However, it requires decontamination of the sample, low temperature $\left(28\right.$ to $\left.33{ }^{\circ} \mathrm{C}\right)$, dark conditions, and $5 \% \mathrm{CO}_{2}$ supplementation, as well as a prolonged period of observation. All those conditions make culture methodology difficult to use in places where the disease is more prevalent. The sensitivity of the culture method is no more than $50 \%$ and mostly useful for follow up and response to therapy $[8,11]$.

The best and faster method of diagnosis is the polymerase chain reaction (PCR), which can give positive results in no more than 2 weeks. Substrates that can be used include fresh and paraffin embedded tissue biopsy samples and cultured isolated material. The easy approach is to detect specific material by PCR: PCR sensitivity and specificity is high (>90\%) when IS2404 is used as a target sequence. Several methods have been described, but quantitative real-time PCR is the most commonly used today for differential diagnosis and environmental studies [8]. However, in the rural setting, diagnosis is commonly made based on clinical findings, and a skin biopsy adequately sent in formalin media can still be quite useful in contributing to the diagnosis

\section{Prognosis}

Since the introduction of medical therapy in 2012 by the WHO, the management of Buruli ulcer has significantly changed. Before 2012, the only therapeutic option available was surgical debridement and amputation, but now early cases can be easily treated with oral antibiotic regimens, avoiding future incapacities to patients. The distinctive histopathological findings in Buruli ulcer make the skin biopsy an interesting tool, readily accessible in any setting, allowing an early diagnosis and establishing an early medical therapy.

\section{A granulomatous lesion on the central face}

Granulomatous disease of the central face is often encountered in general pathology. Whereas in the developed world noninfectious processes tend to predominate, including rosacea and sarcoidosis, in the developing world the differential diagnosis is more ample, on the basis of infectious agents, such as tuberculosis, leishmaniasis, and subcutaneous and systemic mycosis.

A new infectious agent producing a granulomatous process with special tendency to affect the central face has been described in recent years. Although still rare, the infection has been described in developed and developing countries. More significantly, it has the potential for dissemination to the CNS, carrying a great risk of mortality. We will discuss the clinical and pathology aspects of the 
infection by Balamuthia mandrillaris, an environmental free-living ameba with the potential to cause a granulomatous subacute meningoencephalitis associated with a high mortality rate.

\section{Balamuthia mandrillaris infection and its cutaneous manifestation}

\section{Introduction}

Balamuthia mandrillaris belong to the group of free-living amebas capable of inducing CNS involvement; they are commonly associated with delayed diagnosis and high mortality [16]. Other members of the group include Naegleria sp. and Acanthoameba sp. The disease caused by Naeglaria is a primary amebic encephalitis in immunocompetent patients, without any described cutaneous involvement. Acanthamoeba sp. are known to cause a subacute granulomatous meningoencephalitis in immunosuppressed patients with occasional cutaneous manifestations as well as different forms of eye involvement. Balamuthia, instead, has a special characteristic: it can present initially with a skin lesion that is clinically recognizable, allowing for the early diagnosis of a disease with otherwise difficult diagnosis and very high mortality. The clinical lesion is mostly accompanied by a granulomatous infiltrate. The clinical-pathological correlation in an early stage when the disease is limited to the skin may allow for an early diagnosis and the possibility of survival in an otherwise extremely fatal disease $[16,17]$.

\section{Epidemiology and etiopathogenesis}

Balamuthia mandrillaris was initially described as a cause of subacute meningoencephalitis affecting a pregnant female baboon at the San Diego Zoo. It was initially separated from the Acanthamoeba group but classified under the leptomyxid group. In 1993, based on morphology, physiology, and antigens, it was renamed Balamuthia mandrillaris, and although similar to Acanthamoeba in several aspects, it was established as a different genus and species, with its own clinical characteristics. Two cycle stages are currently recognized: the trophozoite and the cyst. The first one is the pathogenic stage. Balamuthia is not able to growth on a plate covered by bacteria, but requires a plate covered with mammalian cells [16]. The ameba does exist in nature, but the natural niche has not been well defined. In contrast with Naegleria and Acanthamoeba, the disease is not always acquired from water sources, but most commonly from the soil. The disease is acquired via the respiratory system or through skin injury; the dissemination does occur via hematogenous spreading, which explains how initial skin lesions may be located distally, beyond the face.

In the last review of cases from the Center for Disease Control and Prevention, Atlanta, Georgia, (CDC), a total of 109 cases of $B$. mandrillaris have been reported in the US in the period between 1974 and 2016 [16]. The Peruvian series of cases at the Instituto de Medicina Tropical Alexander von Humboldt, Universidad Peruana Cayetano Heredia of Lima, Peru, now reach more than 50 cases since 1990 [17]. Both the US and Peruvian group of patients are quite similar with one exception: cutaneous involvement occurs in around $5 \%$ of US patients, but is present in $90 \%$ of Peruvian cases. A preference for the infection in people of Hispanic descent has been demonstrated in studies of Balamuthia encephalitis in California. One can hypothesize that such differences may be explained not only by the pathogenesis of the ameba species, but also by the genetic predisposition to react locally at cutaneous level to delay the dissemination of disease. The disease seems to affect other organs beyond skin and CNS; this is suggested by the fact that transplanted organs such as the kidney may transmit the infection to the recipients [18]. A recent case with a fatal outcome demonstrated the dissemination of the infection to the kidney by autopsy [19]. The real severity of the disease is caused by the dissemination to the CNS, where necrosis of neural tissue and vascular involvement are a common cause of mortality.

It is important to mention that a cutaneous lesion may clinically resolve with therapy; however, the CNS disease may manifest later despite the cutaneous improvement.

\section{Clinical findings}

The cutaneous lesion of Balamuthia infection is usually an asymptomatic plaque. It may look like a well-defined plaque (Fig. 9), even with slightly elevated borders, but occasionally the lesion may look like an area of infiltrative process with somewhat ill-defined borders. The latter is more common in long standing disease affecting the central face and the nose.

The most common location is over the nose, either central or lateralized to any side. The diameter goes from few centimeters to large lesions involving half a face. The color is pink; there is no tenderness, and sensitivity is preserved. Ulceration is rare. The second most common location is the knee area, but lesions have been seen on the buttocks, the thighs, the arms, and the chest. The most common situation is one single lesion but satellites lesions may be seen on face and knee, although never in a sporotrichoid pattern.

"Metastatic" or disseminated cutaneous lesions have been seen in patients that initially had a good clinical 


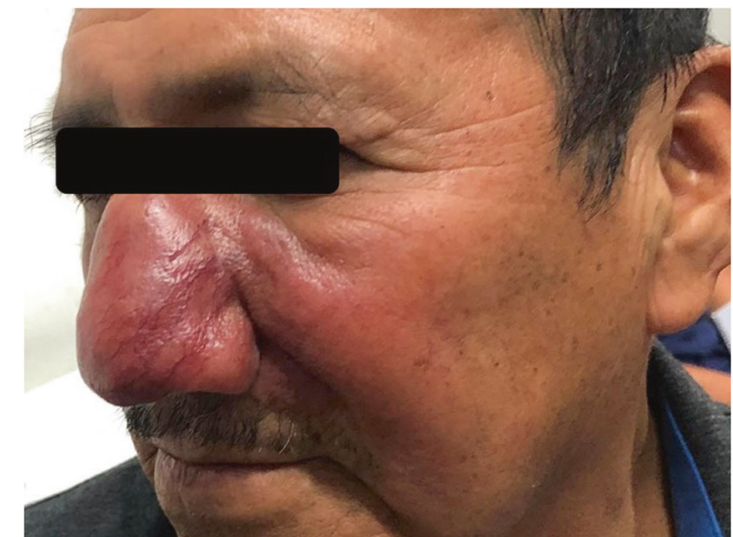

Fig. 9 A plaque lesion over the central face, the typical lesion seen in cutaneous Balamuthiasis

response of the cutaneous lesions to therapy, but then started to develop CNS involvement.

A good initial clinical response to therapy of the cutaneous lesion is by no means a guarantee that the disease is under control. This is the reason why therapy should be prolonged for long periods of time, beyond the complete resolution of the skin lesion.

The manifestations of CNS involvement have an ominous significance. Such manifestations can start as changes in conduct (childish behavior), focal motor deficits, fever, meningeal signs, seizures, and an altered state of consciousness. It is recommended to evaluate the neurological state by imaging even in a neurologically asymptomatic patient, to recognize early asymptomatic affectation of the CNS.

\section{Histopathology}

The cutaneous involvement by Balamuthia includes a diffuse inflammatory infiltrate affecting the reticular dermis. The infiltrate includes a background of lymphocytes and plasma cells, with ill-defined granulomas of epithelioid histiocytes and several to abundant multinucleated giant cells, located inside the granulomas but also interstitially. Collections of neutrophils can be seen occasionally, as well as some eosinophils and foci of necrosis.

The trophozoites measure $15-30 \mu \mathrm{m}$, where the cysts measure 10-30 $\mu \mathrm{m}$. Cysts are seen in the brain but not in the skin. Trophozoites can be seen in $70 \%$ of cases in the skin biopsy, but they are scarce and easily confused with histiocytes [20]. To tell them apart, one should look especially at the nucleus and nucleolus. The shape of the trophozoite could be as expected, with a jagged border, but also oval shaped (Figs. 10 and 11). The cytoplasm can have a bubbly appearance (Fig. 11). The whole trophozoite may be situated interstitially, encased in an empty space or inside a giant cell. When abundant, they can be located in the wall of vessels of

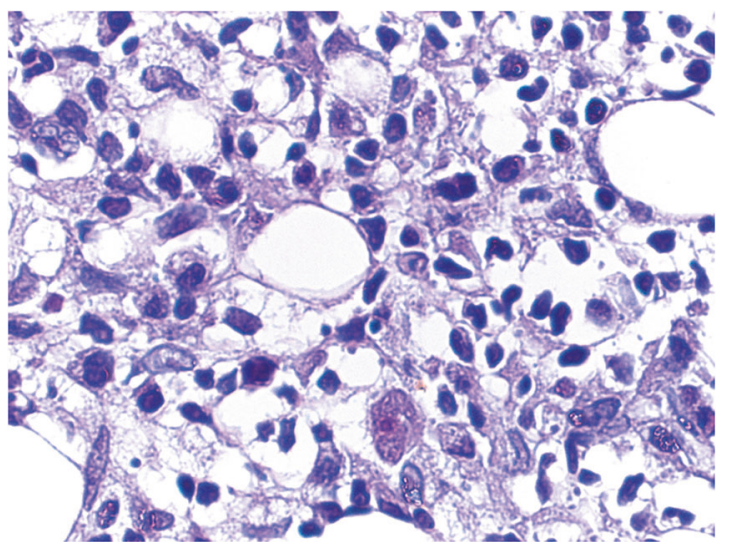

Fig. 10 A trophozoite of $B$. mandrillaris with more of a classical cytoplasm. This type can be easily confused with a histiocytes if the nucleus is not seen in the section. $\mathrm{H} / \mathrm{E}, \times 1000$

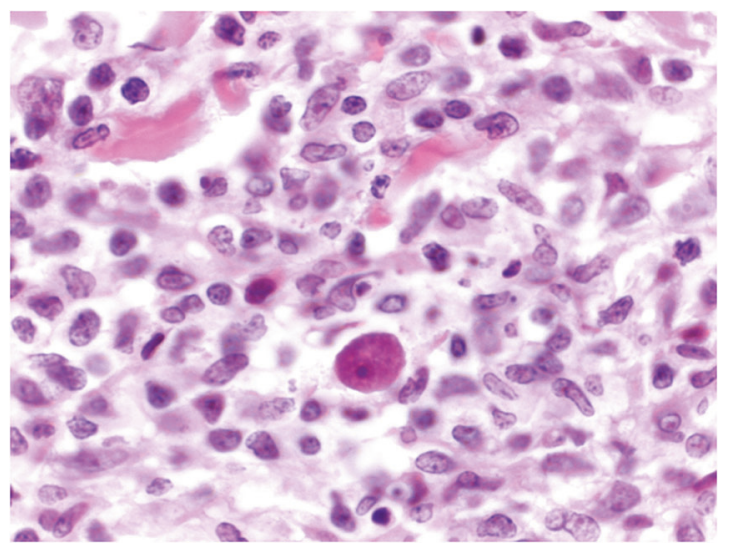

Fig. 11 A Balamuthia mandrillaris trophozoite with oval shaped and bubbly cytoplasm. H/E, $\times 1000$

small caliber and may be confused with enlarged histiocytes or endothelial cells. The key is to recognize the nucleus, which is completely different from that of a human cell (Fig. 10). However, depending on the level of the pathology section, the trophozoite may be confused for histiocytes. Identification of the trophozoite may require deeper cuts and repeated observation of the slide. At this point, early identification of the infective agent may save the patient's life.

No particular routine stain has been found to make a difference besides $\mathrm{H} / \mathrm{E}$, but direct immunofluorescence testing and inmunoperoxidase techniques as developed by the CDC are quite sensitive and specific [21].

With regard to molecular studies for detection of the amebic DNA material in human tissue, the most recent method provided by the $\mathrm{CDC}$, is real-time PCR targeting the RNase $\mathrm{P}$ gene.

To get the CDC's cooperation it is highly recommended to contact them by the DPDx service (https://www.cdc.gov/ dpdx/index.html). Direct examination and culture from 
CNS fluid and tissue also can be done when the disease already involve the CNS.

\section{Diagnosis}

The diagnosis of Balamuthia mandrillaris at a cutaneous level is critical. It does require a compatible clinical context as well a compatible histological pattern. Identifying the microorganism requires some degree of expertize. If the diagnostic possibility of Balamuthia infection exists, one should not hesitate to contact a reference laboratory and establish an early therapy.

Differential diagnosis should include other infectious processes, including tuberculosis, leishmaniasis, leprosy, subcutaneous, and systemic mycosis, non-infectious processes such as granulomatosis with angeitis, sarcoidosis, and some neoplastic processes, including Nasal type of NK$\mathrm{T}$ cell Lymphoma.

\section{Prognosis}

Without therapy, the infection is associated with extremely high mortality, even in cases where the disease may look like it is limited to the skin, because the CNS involvement could still be clinically silent. The presence of CNS disease makes the prognosis definitively worse. Great effort should be directed towards establishing an early diagnosis. There now exists a possibility of medical therapy in patients either with isolated cutaneous involvement or already with involvement of CNS, using a combination of drugs including an antiparasitic medication called miltefosin [22]. Even patients with neurological involvement may have some chance of surviving if therapy is initiated early.

\section{Compliance with ethical standards}

Conflict of interest The authors declare that they have no conflict of interest.

Publisher's note Springer Nature remains neutral with regard to jurisdictional claims in published maps and institutional affiliations.

\section{References}

1. Herman JS, Chiodini PL. Gnathostomiasis, another emerging imported disease. Clin Microbiol Rev. 2009;22:484-92.

2. Bravo F, Gontijo B. Gnathostomiasis: an emerging infectious disease relevant to all dermatologists. An Bras Dermatol. 2018;93:172-180.
3. Neumayr A, Ollague J, Bravo F, et al. Cross-reactivity pattern of Asian and American human gnathostomiasis in Western Blot assays using crude antigens prepared from Gnathostoma spinigerum and Gnathostoma binucleatum third-stage larvae. Am J Trop Med Hyg. 2016;95:413-6.

4. Diaz JH. Gnathostomiasis: an emerging infection of raw fish consumers in Gnathostoma nematode-endemic and nonendemic countries. J Travel Med. 2015;22:318-24.

5. Leroy J, Cornu M, Deleplancque AS, et al. Sushi, ceviche and gnathostomiasis - a case report and review of imported infections. Travel Med Infect Dis. 2017;20:26-30.

6. Alvarez P, Morales A, Bravo F. Gnathostomiasis: experiencia en una práctica privada en Lima, Peru. Folia Dermatológica Peru. 2011;22:67-74.

7. Laga AC, Lezcano C, Ramos C, et al. Cutaneous gnathostomiasis: report of 6 cases with emphasis on histopathological demonstration of the larva. J Am Acad Dermatol. 2013;68:301-5.

8. Yotsu RR, Suzuki K, Simmonds RE, et al. Buruli ulcer: a review of the current knowledge. Curr Trop Med Rep. 2018;5:247-56.

9. Guerra H, Palomino JC, Falconí E, et al. Mycobacterium ulcerans disease, Peru. Emerg Infect Dis. 2008;14:373-7.

10. Guarner J, Bartlett J, Whitney EA, et al. Histopathologic features of Mycobacterium ulcerans infection. Emerg Infect Dis. 2003;9:651-56.

11. Guarner J. Buruli Ulcer: review of a neglected skin Mycobacterial disease. J Clin Microbiol. 2018;56:1-8.

12. Lindo SD, Daniels F Jr. Buruli ulcer in New York City. J Am Med Assoc. 1974;228:1138-9.

13. Semret M, Koromihis G, MacLean JD, et al. Mycobacterium ulcerans infection (Buruli ulcer): first reported case in a traveler. Am J Trop Med Hyg. 1999;61:689-93.

14. Faber WR, de Jong B, de Vries HJ, et al. Buruli ulcer in traveler from Suriname, South America, to the Netherlands. Emerg Infect Dis. 2015;21:497-9.

15. Guerra Laso JM, Nebreda Mayoral T, Palacios Gutiérrez JJ, et al. Buruli ulcers in a Spanish aid worker after a stay in Peru. Int $\mathrm{J}$ Infect Dis. 2019;78:99-102.

16. Cope JR, Landa J, Nethercut $\mathrm{H}$, et al. The epidemiology and clinical features of Balamuthia mandrillaris disease in the United States, 1974-2016. Clin Infect Dis. 2019;68:1815-22.

17. Bravo FG, Seas C. Balamuthia mandrillaris amoebic encephalitis: an emerging parasitic infection. Curr Infect Dis Rep. 2012;14:391-6.

18. Farnon EC, Kokko KE, Budge PJ, et al. Transmission of Balamuthia mandrillaris by organ transplantation. Clin Infect Dis. 2016;63:878-88.

19. Shehab KW, Aboul-Nasr K, Elliott SP. Balamuthia mandrillaris Granulomatous Amebic Encephalitis With Renal Dissemination in a Previously Healthy Child: Case Report and Review of the Pediatric Literature. J Pediatr Infect Dis Soc. 2018;7:e163-e168.

20. Sangueza O, Bravo Puccio F, Sangueza JM. Diseases caused by Amebas in: dermatopathology of tropical diseases: pathology and clinical correlations. London: JP medical publisher;2017.

21. Guarner J, Bartlett J, Shieh WJ, Paddock CD, Visvesvara GS, Zaki SR. Histopathologic spectrum and immunohistochemical diagnosis of amebic meningoencephalitis. Mod Pathol. 2007;20:1230-7.

22. Martínez DY, Seas C, Bravo F, et al. Successful treatment of Balamuthia mandrillaris amoebic infection with extensive neurological and cutaneous involvement. Clin Infect Dis. 2010;51: e7-11. 